\title{
Synthesis of PVA Encapsulated Silver Nanoparticles as a Drug Delivery System for Doxorubicin and Curcumin
}

\author{
Nhi Nguyen, Cam Huong Le \\ 53 Nguyen Du, Dist. 1, Ho Chi Minh City, 700000, Vietnam ; nhituyet6788@gmail.com
}

\begin{abstract}
Silver nanoparticles, of great significance in drug delivery, are unstable due to agglomeration. To address this problem, we examined the effectiveness of polyvinyl alcohol (PVA) as a cheap, environmentally-friendly biomaterial in stabilizing and increasing the efficiency of drug-loaded silver nanoparticles. In this study, we have successfully synthesized in situ PVA-coated nanoparticles with a particle size of $70.1 \mathrm{~nm}$ measured by DLS and $46.7 \mathrm{~nm}$ measured using SEM by chemical reduction method using silver nitrate $\left(\mathrm{AgNO}_{3}\right)$, sodium borohydride $\left(\mathrm{NaBH}_{4}\right)$, and PVA. Drug loading efficiency of AgNPs-PVA reached $91.95 \%$ for Doxorubicin and $99.24 \%$ for Curcumin, which is higher than the drug loading efficiency of uncoated silver nanoparticles. Additionally, we have determined the antibacterial activity of AgNPs-PVA and AgNPs-PVA-Cur on strains of gram (+) Bacillus cereus and gram (-) E. coli and a concentration of $5 \mathrm{ppm}$ and above yield zones of inhibition greater than $10 \mathrm{~mm}$ in diameter. Given time and additional equipment, we hope to conduct further studies that examine the anticancer properties of AgNPs-PVA-Dox on cancer cells and the burn treatment applications of AgNPs-PVA-Cur on mice.

KEYWORDS: Nanomedicine; Drug Loading; PVA-coated Silver Nanoparticles; Breast Cancer Therapeutics; Burns Treatment.
\end{abstract}

\section{- Introduction}

Over the past decades, silver nanoparticles have attracted great attention from scientists around the world due to their unique properties and wide applicability. Specifically, silver nanoparticles have been studied and used as catalysts ${ }^{1}$ and biological probes. ${ }^{2}$ In particular, thanks to their effective antimicrobial activity, ${ }^{3,4}$ silver nanoparticles are believed to be a potential solution to the problem of biological infection.

Throughout the world, the production of silver nanoparticles has been carried out by different methods such as chemical reduction methods, physical reduction methods, biological reduction methods, etc. The FM Gutierrez team used chemical agents to reduce silver ions to silver; the resulting silver nanoparticles were smaller than $100 \mathrm{~nm} .{ }^{5} \mathrm{H}$. Jiang et al. used high-energy electromagnetic waves such as gamma rays, ultraviolet rays, and lasers to synthesize silver nanoparticles. ${ }^{6}$ Some studies used chemical reducing agents such as sodium borohydride, ${ }^{7}$ ascorbic acid ${ }^{8}$ to reduce silver ions to silver nanoparticles. In this study, we employed a chemical reduction method using sodium borohydride as a reducing agent because the method is the least time-consuming and requires minimal laboratory equipment.

Doxorubicin (Dox) is an active ingredient that is commonly used in breast cancer treatment today. However, Dox causes many serious side effects. Treatment with doxorubicin often leads to undesirable reactions that need to be closely monitored. Undesirable effects often depend on the route of administration, dosage, administration's speed and frequency. Nausea, vomiting and hair loss are common in many patients. Impaired bone marrow function is a very common adverse reaction to dose limits. One more side effect worth noting is the accumulation of cardiotoxins in the patient's body. ${ }^{9}$ Silver nanoparticles in drug delivery, especially for Doxorubicin as a breast cancer treatment, are of great significance in the biochemistry field for their ability to bypass the cell membrane via endocytosis and to localize into the lysosomes. Here, the NPs release the drug directly, damaging cell's DNA and lead to cell death. ${ }^{10}$ Thus, AgNPs lead the drug to its destination, reducing dosage and side effects. ${ }^{11}$

Curcumin is a bright yellow chemical produced by Curcu$m a$ longa plants. It is the principal curcuminoid of turmeric (Curcuma longa), a member of the ginger family, Zingiberaceae. Research by scientists in the late twentieth century has determined that curcumin plays an important role in the biological activities of turmeric. Based on in vitro and animal studies, scientists have proven that curcumin has antibacterial and anti-scar wound healing properties. ${ }^{12,13}$

Burns are conditions of the skin due to heat damage which are very common in real life. First degree burns result in pain and reddening of the epidermis (outer layer of the skin) while second-degree burns (partial thickness burns) affect the epidermis and the dermis (lower layer of skin, causing pain, redness, swelling, and blistering which can lead to infection. At the nanoscale, silver increases its antiseptic activity by up to 50000 times greater compared with that of silver in ionic size. Silver nanoparticles can continually release silver ions and owing to electrostatic attraction and affinity to sulfur proteins, silver ions can adhere to the cell wall and cytoplasmic membrane. The adhered ions can enhance the permeability of the cytoplasmic membrane and lead to disruption of the bacterial envelope. ${ }^{13}$ Therefore, the use of silver nanoparticles in combination with Curcumin with its antibacterial and anti-scar wound healing 
properties is also seen as a potential approach in treating burns, which are easily infected and result in severe skin scarring.

In order for nanoparticles to disperse well in the solvent without agglomeration, a coating with a surfactant or an electrostatic method that causes the nanoparticles' surface to have the same charge and repel each other is used. ${ }^{14}$ Nanoparticles formed by these methods are usually less than $100 \mathrm{~nm}$ in size. In this study, we examined the effectiveness of PVA as a biocompatible, water-soluble, inexpensive and nontoxic surfactant in stabilizing and increasing the efficiency of drug-loaded silver nanoparticles. ${ }^{15}$ While Doxorubicin-loaded and Curcumin-loaded nanoparticles have been previously studied such as that of Y. Du et al. on Doxorubicin-loaded gold nanoparticles ${ }^{16}$ and S. Ravindra et al. on Curcumin-loaded silver nanoparticles hydrogels ${ }^{17}$ and PVA has previously been used in combination with silver nanoparticles as a stabilizer, ${ }^{18}$ using PVA to stabilize and increase the efficiency of silver nanoparticles for loading and delivering doxorubicin and curcumin specifically is new. The first goal of our study is, by chemical reduction methods, to develop PVA-coated silver nanoparticle (AgNPs-PVA) synthesis process with $\mathrm{NaBH}_{4}$ as the reducing agent and determine the physicochemical properties of silver nanoparticles that formed: structure, particle size, and distribution. Next, we compared the drug loading effectiveness of AgNPs-PVA for Doxorubicin and Curcumin to uncoated silver nanoparticles. The ultimate goal is to test anticancer properties of Doxorubicin-loaded AgNPsPVA (AgNPs-PVA-Dox) on cancer cells, and antibacterial properties of Curcumin-loaded AgNPs-PVA (AgNPs-PVACur) on mice and implement them for treatments on humans.

\section{- Result and Discussion \\ Analysis of Physicochemical Properties:}

In this study, we proceeded to coat silver nanoparticles with polyvinyl alcohol by in situ method, which means that $\mathrm{Ag}-$ NPs-PVA nanoparticle synthesis process is in parallel with AgNPs granulation process.

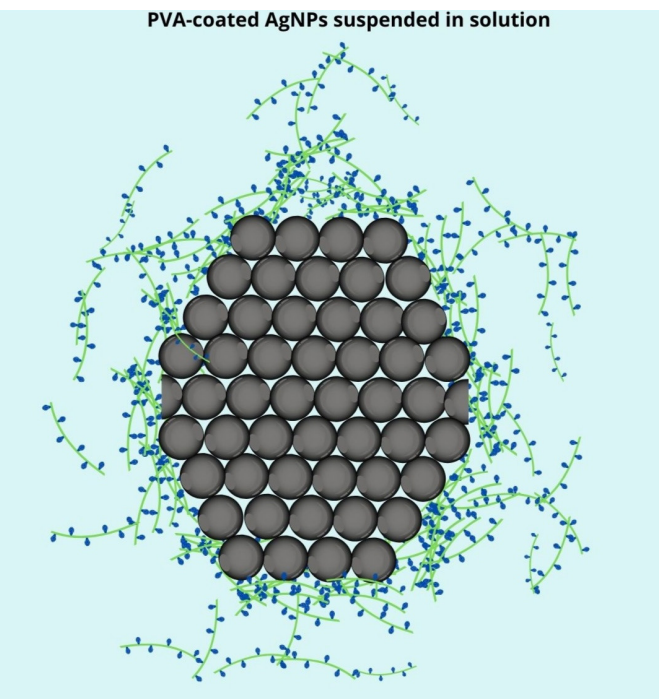

Figure 2.1: Structural properties of AgNPs-PVA.
One of the roles of PVA as a coating agent in the synthesis of silver nanoparticles is to protect them from self-aggregation in solution. Structural flexibility and conformational dynamics of a stabilizing agent backbone or a polymer chain, adsorbed onto a nanoparticle surface, play an important role in colloidal stabilization, shape-controlled growth, as well as the water-protecting effect of silver nanoparticles. ${ }^{19}$ To gain a better understanding of non-bonded interactions, occurring at the organic/inorganic interface between PVA, the AgNP core, and the surrounding water molecules, a scheme of possible interactions is given in Figure 2.1.

When conducting spectroscopy of the samples, each sample had a defined peak spectral height like the standard UVvis spectrum of silver nanoparticles from which the silver nanoparticles could be determined. In this case, the occurrence of silver nanoparticles could be detected if the UV-vis spectrophotometer result showed the absorption peak around wavelengths of $430 \mathrm{~nm}$. Simultaneously, the higher the peak is on the absorbance axis, the more of the light with this particular wavelength is absorbed, indicating higher concentration of silver nanoparticles in aqueous solution. Thus, this property helps determine if the silver nanoparticles synthesis process had happened and make preliminary prediction of the concentration of silver nanoparticles formed. ${ }^{20}$

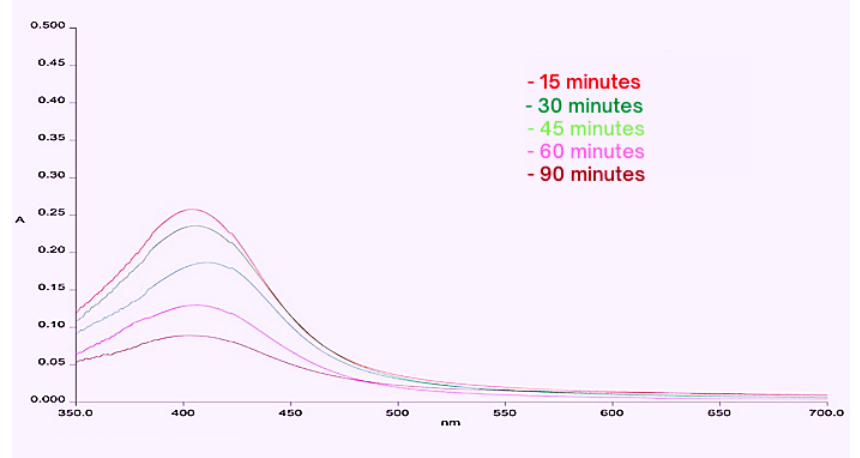

Figure 2.2: UV-Vis spectra of AgNPs-PVA solution over stirring time of 15 minutes, 30 minutes, 45 minutes, 60 minutes, and 90 minutes.

Based on this ground work, we varied the stirring time of each batch from 15 to 90 minutes in order to examine the occurrence and concentration of our silver nanoparticles suspended in water at room temperature. We measured each batch with a UV-vis spectrophotometer, whose spectrum range was set from $350 \mathrm{~nm}$ to $700 \mathrm{~nm}$, and obtained absorption peaks at 400 to $409 \mathrm{~nm}$ with absorbance of 0.075 to 0.25 . This result indicated that silver nanoparticles were formed although the concentration of nanoparticles in solutions was quite low. However, the most optimal stirring was determined at 15

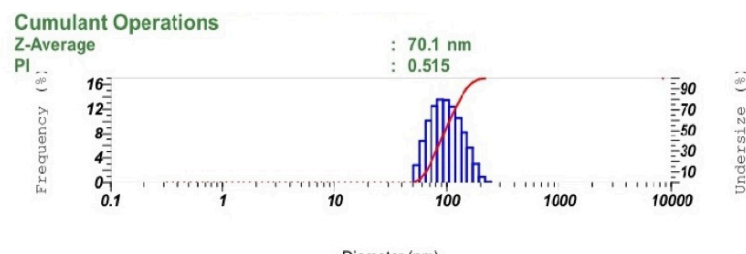

Figure 2.3: Particle size distribution of AgNPs-PVA measured by DLS. 
minutes, which yielded the maximum peak wavelength at 409 $\mathrm{nm}$ and highest absorbance at 0.25 (Figure 2.3).

With the formation of silver nanoparticles confirmed and optimal stirring time determined, we synthesized more batches and sent them to Academy of Chemical Engineering-Vietnam Academy of Science and Technology. Our aim was to measure the particle size distribution AgNPs-PVA by dynamic light scattering (DLS), which determines a relaxation time for the decay of the autocorrelation function of the scattered light. From that, a diffusion coefficient inversely proportional to particle size can be extracted. Results of the size distribution of AgNPs-PVA nanoparticles by DLS showed that the nanoparticles formed were, in average, approximately $70.1 \mathrm{~nm}$ in size (Figure 2.5).

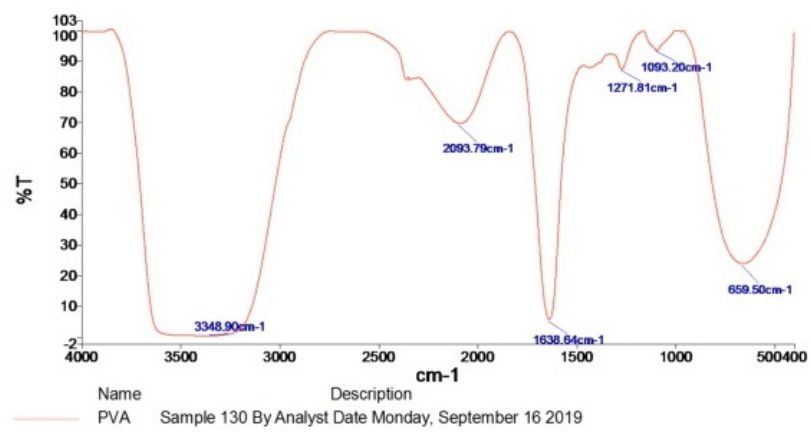

Figure 2.4: FTIR spectra of AgNPs-PVA nanoparticles.

Consequently, to confirm that the silver nanoparticles have been coated with PVA, we conducted analysis of AgNPs-PVA by FTIR spectroscopy at wavenumbers $4000-400 \mathrm{~cm}^{-1}$. Figure 2.4 shows that FTIR spectrum of AgNPs-PVA appears to have characteristic peaks at wavenumbers $3348 \mathrm{~cm}^{-1}, 1638$ $\mathrm{cm}^{-1}, 659 \mathrm{~cm}^{-1}$, which corresponds to the O-H, C-C bond, and the torsional fluctuations of the $\mathrm{OH}$ group. This indicated the presence of PVA coating on the surface of AgNPs and bonds between PVA and AgNPs.

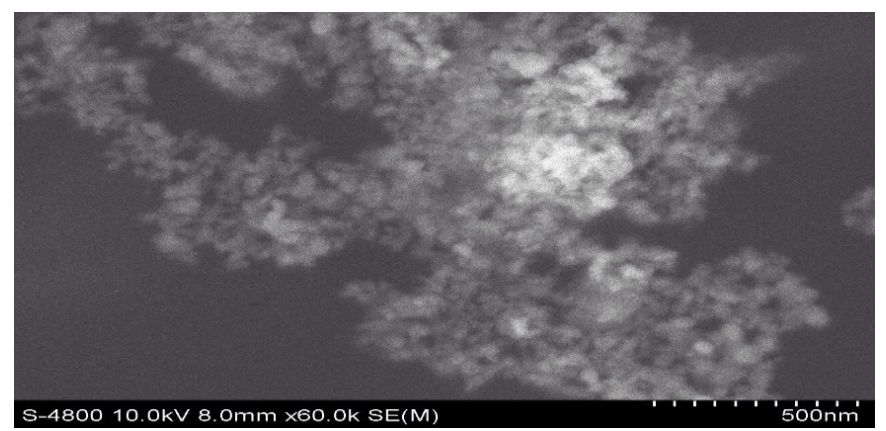

Figure 2.5: SEM measurement results of AgNPs-PVA.

The final method of chemical analysis we employed was scanning electron microscopy (SEM), which scans a focused electron beam over of the nanoparticles. The electrons in the beam interact with the sample, producing various signals that can be used to obtain information about the surface topography and composition. SEM results showed that the AgNPs-PVA formed were spherical (Figure 2.5), the total number of particles was 394 and the average particle size was $46,764 \mathrm{~nm}$
(Table 3.1), which is smaller than the size measured by the DLS (Figure 2.5).

The reason for the difference in the two methods lies in the mechanism of DLS, which does not measure size but, instead, diffusivities of AgNPs-PVA in an aqueous solution. If given monodisperse size distribution, i.e. all particles of the same size, then a size measurement by SEM should give a size similar to that measured by DLS. If a distribution of particle sizes exists in a solution, DLS weights the distribution differently compared to SEM, by size to the power of 6, essentially giving a $\mathrm{z}$-average distribution. Larger particles are, therefore, given more weight, making the "average" size look larger than SEM's. Hence, the SEM result is a more reliable indicator of AgNPs-PVA size.

\section{Drug Loading Efficiency of AgNPs-PVA:}

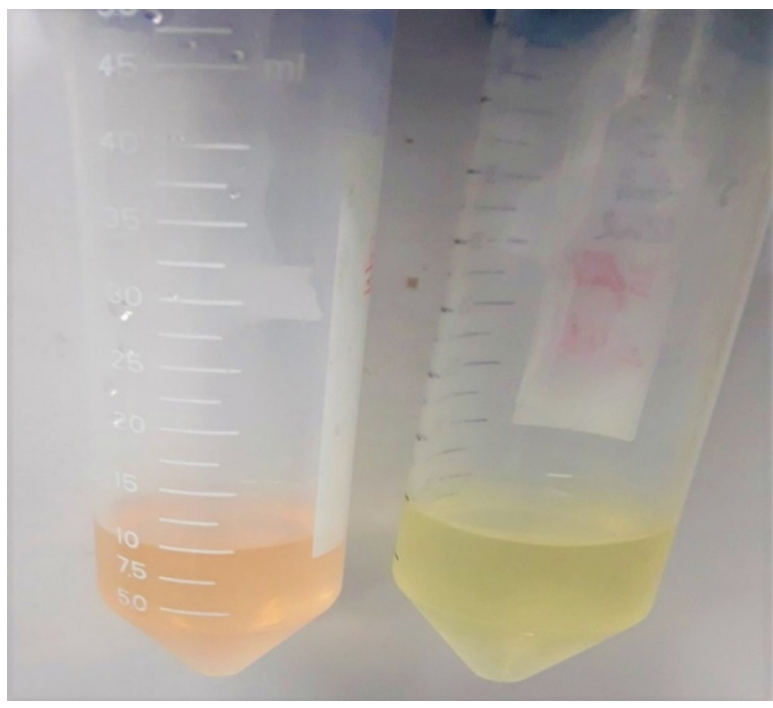

Figure 2.6: AgNPs-PVA-Dox solution (left), AgNPs-PVA-Cur (right).

We proceeded to load Doxorubicin and Curcumin onto AgNPs-PVA with concentration of 200 ppm by shaking and centrifuge. Based on Figure 2.1 in which the solid silver core is coated with dispersed PVA particles, the drug is expected to coat and be absorbed on the PVA surface of the AgNPs-PVA.

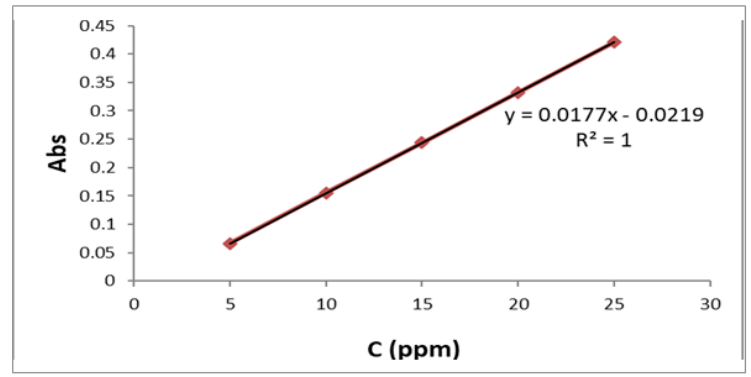

Figure 2.7: Calibration curve showing the relationship between concentration and loading efficiency of Doxorubicin.

A standard calibration curve was constructed for AgNPSPVA-Dox and AgNPs-PVA-Cur in order to obtain the linear equation which was further used to calculate the drug-loading efficiency. We prepared stock Doxorubicin and Curcumin solutions with a concentration of $100 \mathrm{ppm}$. From these two 
stock solutions, suitable dilutions were prepared at $5 \mathrm{ppm}, 10$ ppm, 15 ppm, $20 \mathrm{ppm}$, and $25 \mathrm{ppm}$. All the dilutions were analyzed by UV-Visible Spectrophotometer. The values of absorbance were then recorded and a standard curve was constructed by plotting absorbance against concentration. From this curve a regression line equation was obtained in $\mathrm{MS} \mathrm{Ex-}$ cel format.

Once the calibration curve for Doxorubicin was obtained, we analyzed our 200 ppm AgNPs-PVA-Dox, which was synthesized beforehand, by UV-Visible Spectrophotometer. The absorbance was recorded at 0.2632 , which was then plotted into the equation of the calibration curve to calculate the unloaded Doxorubicin amount of 16.2090 ppm. This experimental result showed that Doxorubicin drug loading efficiency of AgNPs-PVA reached 91.9455\% (Figure 2.7).

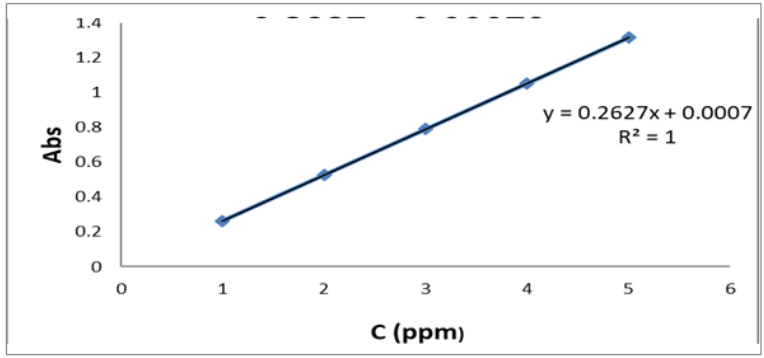

Figure 2.8: Calibration curve showing the relationship between concentration and loading efficiency of Curcumin.

Once the calibration curve for Curcumin was obtained, we analyzed our 200 ppm AgNPs-PVA-Cur, which was synthesized beforehand, by UV-Visible Spectrophotometer. The absorbance was recorded at 1.9679 , which was then plotted into the equation of the calibration curve to calculate the unloaded Curcumin amount of $7.4883 \mathrm{ppm}$. This experimental result showed that Curcumin drug loading efficiency of $\mathrm{Ag}-$ NPs-PVA reached 96.2559\% (Figure 2.8).

\section{Antibacterial Properties of AgNPs-PVA and AgNPs-PVA-Cur:}
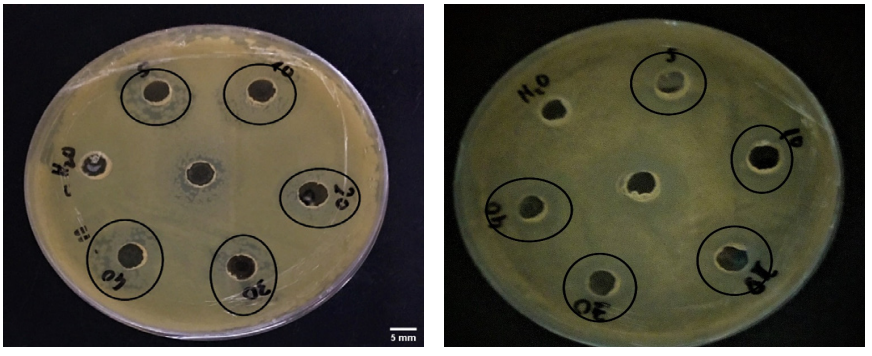

a) Bacteria Bacillus cereus (gram positive)

b) E. coli bacteria (gram negative)

Figure 2.9: Petri dishes showing zones of inhibition against bacteria Bacillus cereus (gram positive) and $E$. coli bacteria (gram negative) for AgNPsPVA concentrations of 5 ppm, 10 ppm, 20 ppm, 30 ppm, 40 ppm.

The experimental results showed that AgNPs-PVA became antibacterial at the concentration of $5 \mathrm{ppm}$. At a concentration of $5 \mathrm{ppm}$, diameters of the zones of inhibition of AgNPs-PVA in both bacteria were greater than $10 \mathrm{~mm}$. The greatest diameters recorded were $20 \mathrm{~mm}$ from the concentration of $40 \mathrm{ppm}$ for Bacillus cereus and $20 \mathrm{~mm}$ from the concentration of $40 \mathrm{ppm}$ for $E$. coli. This confirms the antibacterial potential of AgNPs-PVA (Figure 2.9).

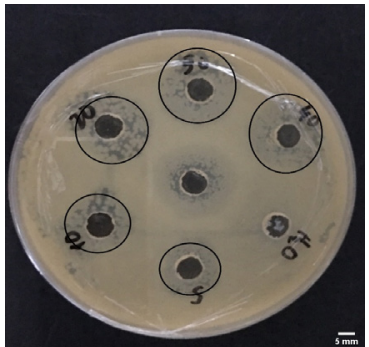

a) Bacteria Bacillus cereus (gram positive)

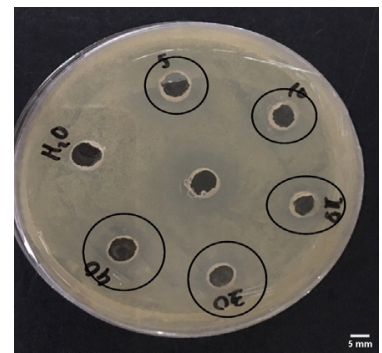

b) E. coli bacteria (gram negative)
Figure 2.10: Petri dishes showing zones of inhibition against bacteria Bacillus cereus (gram positive) and $E$. coli bacteria (gram negative) for AgNPsPVA concentrations of 5 ppm, 10 ppm, 20 ppm, 30 ppm, 40 ppm.

The experimental results showed that AgNPs-PVA-Cur became antibacterial at a concentration of $5 \mathrm{ppm}$. At a concentration of $5 \mathrm{ppm}$, the zone of inhibition of AgNPs-PVA-Cur is greater than $15 \mathrm{~mm}$. The greatest diameters recorded were $23 \mathrm{~mm}$ from the concentration of $40 \mathrm{ppm}$ for Bacillus cereus and $21 \mathrm{~mm}$ from the concentration of $40 \mathrm{ppm}$ for $E$. coli This confirms the antibacterial potential of AgNPs-PVA-Cur and shows an improvement in antibacterial potential of AgNPsPVA-Cur when compared with AgNPs-PVA (Figure 2.10).

\section{- Conclusion}

In this study, we have successfully synthesized AgNPs-PVA with particle size of $70.1 \mathrm{~nm}$ measured by DLS and $46.7 \mathrm{~nm}$ measured by SEM. We proceeded to examine the drug loading efficiency of AgNPs-PVA for Doxorubicin and Curcumin, which reached $91.9455 \%$ and $99.2411 \%$, respectively. We have tested the antibacterial activity of AgNPs-PVA and AgNPsPVA-Cur on gram (+) Bacillus cereus and gram (-) E. coli for concentrations of 5 ppm, 10 ppm, 20 ppm, 30 ppm, 40 ppm and found that AgNPs-PVA and AgNPs-PVA-Cur become antibacterial at the concentration of $5 \mathrm{ppm}$ and above. Hence, AgNPs-PVA-Dox was found to have the potential to be tested on breast cancer cells and be implement into breast cancer therapeutics due to their high drug loading efficiency. Furthermore, AgNPs-PVA-Cur was found to have the potential to be tested on burns spots on mice due to their antibacterial activity.

\section{- Methods}

\section{Materails:}

Chemicals included silver nitrate $\left(\mathrm{AgNO}_{3}\right)$, sodium borohydride $\left(\mathrm{NaBH}_{4}\right)$, and polyvinyl alcohol (PVA) obtained from Scharlau Chemicals (Spain), Curcumin from Ho Chi Minh City University of Agriculture and Forestry, and Doxorubicin hydroclorid $(10 \mathrm{mg} / 5 \mathrm{ml})$ from Fresenius Kabi.

We used gram-positive bacteria Bacillus cereus, gram-negative E. coli from Ho Chi Minh City University of Natural Sciences to investigate the antibacterial properties of $\mathrm{Ag}$ NPs-PVA and AgNPs-PVA-Cur. ${ }^{6}$

The Process of Synthesizing AgNPs-PVA from $\mathrm{AgNO}_{3}$, $\mathrm{NaBH}_{4}$ :

The silver nanoparticle synthesis process was carried out as follows. A $0.001 \mathrm{M} \mathrm{AgNO} 3$ solution was dripped into $50 \mathrm{ml}$ of $2 \%$ PVA solution. Then, while while the mixture was being 
stirred for 15 minutes in the dark, $0.001 \mathrm{M} \mathrm{NaBH} 4$ was added dropwise until the solution turned pale yellow. The reacted solution was centrifuged at $13000 \mathrm{rpm}$ and washed three times with deionized distilled water. The obtained AgNPs-PVA was stored at $4^{\circ} \mathrm{C} .^{21}$

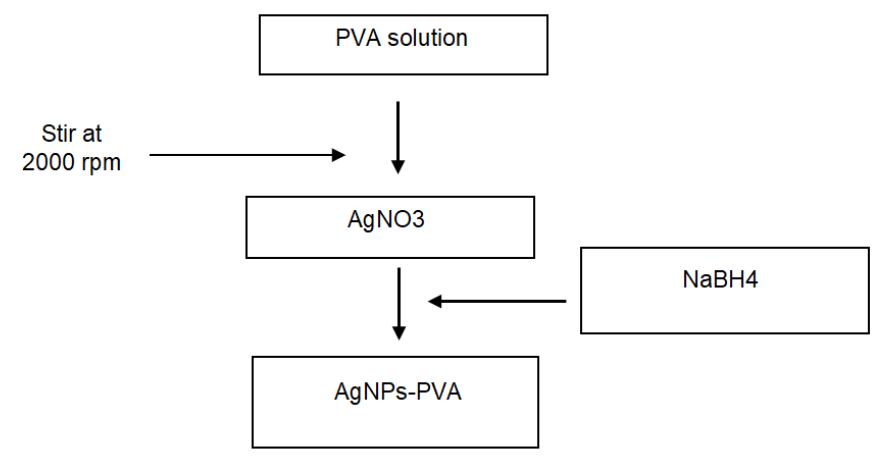

Diagram 1.1: The process of particle synthesis AgNPs- PVA.

The Process of Loading Doxorubicin and Curcumin into AgNPs-PVA:

$1 \mathrm{ml}$ of Dox / Cur solution $(0.2 \mathrm{mg} / \mathrm{mL})$ was added to $9 \mathrm{ml}$ of AgNPs-PVA solution $(2 \mathrm{mg} / \mathrm{ml})$ at $\mathrm{pH}=7.4$. The reaction mixture was shaken with a shaker at temperature $37^{\circ} \mathrm{C}$ for 24 hours in the dark. Then, the reaction mixture was centrifuged for 15 minutes. $^{22}$

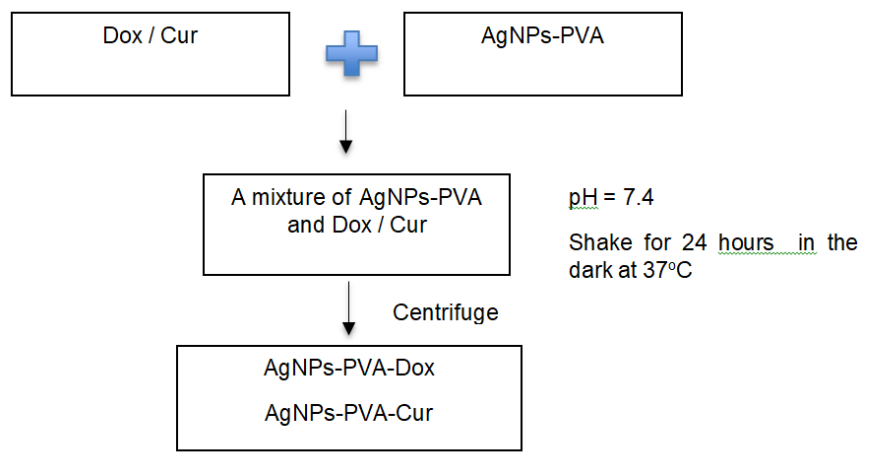

Diagram 1.2: The process of synthesizing AgNPs- PVA-Dox and AgNPsPVA-Cur.

\section{Chemical Analysis Methods:}

Ultraviolet-visible spectroscopy (UV-Vis) is a quantitative analysis method, based on the absorption effect that occurs when matter molecules interact with electromagnetic radiation. The radiation region used in this study is the infrared region with wavelengths from 350 to $700 \mathrm{~nm}$. The phenomenon of electromagnetic radiation absorption complies with the Bouger - Lambert - Beer law. In this study, we used the model UV-Vis Biochrom S60 from Ho Chi Minh City Pedagogical University.

Dynamic light scattering (DLS) is a technique that can be used to determine the size distribution profile of small particles in suspension or polymers in solution. Fourier-transform infrared spectroscopy (FTIR) is a technique used to obtain an infrared spectrum of absorption or emission of a solid, liquid, or gas. Scanning electron microscope (SEM) is a type of electron microscope that produces images of a sample by scanning the surface with a focused beam of electrons. In this study, we sent the silver nanoparticles to Pharmaceutical Chemistry Research Center - Vietnam Academy of Science and Technology to be analyzed by DLS, FTIR, and SEM.

\section{Data Analysis Methods:}

Particle size: we used ImageJ software to analyse the particle size of AgNPs-PVA from SEM results. The count in the section was 394 particles, with total area of 18425 . Therefore, the average size of nanoparticles calculated was $46.764 \mathrm{~nm}$.

Drug loading efficiency:

-A standard calibration curve was constructed for AgNPSPVA-Dox and AgNPs-PVA-Cur in order to obtain the linear equation which was further used to calculate the drug-loading efficiency. For this purpose, stock Doxorubicin and Curcumin solutions were prepared with a concentration of $100 \mathrm{ppm}$. From this stock solution, suitable dilutions were prepared at 5 ppm, 10 ppm, 15 ppm, 20 ppm, and 25 ppm. All the dilutions were analyzed by UV-Visible Spectrophotometer (UV-Vis Biochrom S60). The values of absorbance were recorded and a standard curve was constructed by plotting absorbance against

Table 1: Absorbance values of Doxorubicin solution at $1 \mathrm{ppm}, 2 \mathrm{ppm}, 3$ ppm, 4 ppm, 5 ppm.

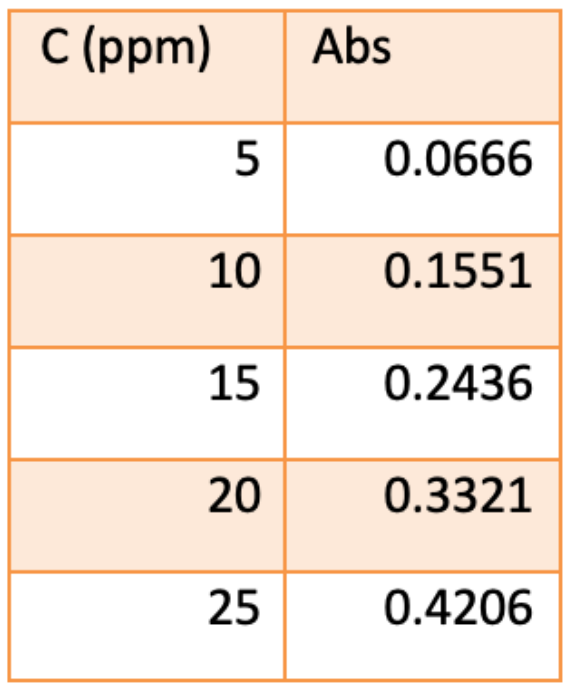

Table 2: Absorbance values of Curcumin solution at $1 \mathrm{ppm}, 2 \mathrm{ppm}, 3 \mathrm{ppm}$, 4 ppm, 5 ppm.

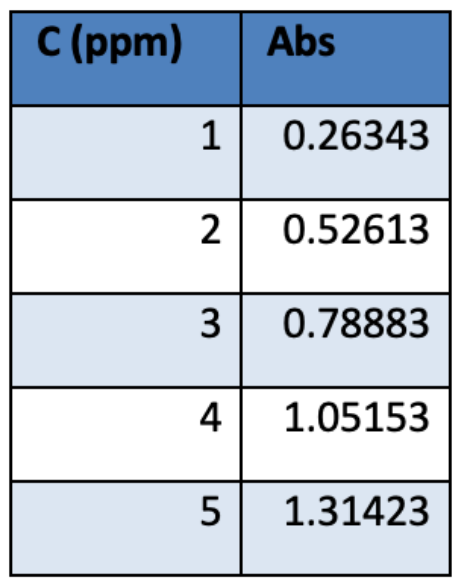


concentration. From this curve a regression line equation was obtained in MS Excel format.

-We proceeded to analyze our 200 ppm AgNPs-PVA-Dox/ Cur, which was synthesized beforehand, by UV-Visible Spectrophotometer. The absorbance was recorded, which was then plotted into the equation of the calibration curve to calculate the unloaded amount of Doxorubicin and Curcumin. Consequently, the loading efficiencies were obtained.

-The efficiency of Doxorubicin loading is calculated by the formula below: ${ }^{23}$

Analyzing antibacterial properties: In order to test $\mathrm{Ag}-$ NPs-PVA and AgNPs-PVA-Cur's antibacterial properties, bacteria Bacillus cereus (gram positive) and E. coli bacteria (gram negative) were cultivated in petri dishes. Next, stock AgNPs-PVA and AgNPs-PVA-Cur solutions were prepared with a concentration of $100 \mathrm{ppm}$. From this stock solution, suitable dilutions were prepared at 5 ppm, 10 ppm, 20 ppm, 30 ppm, and $40 \mathrm{ppm} .10 \mathrm{~g}$ of each solution was injected into the petri dishes with corresponding amount of Doxorubicin and Cur-

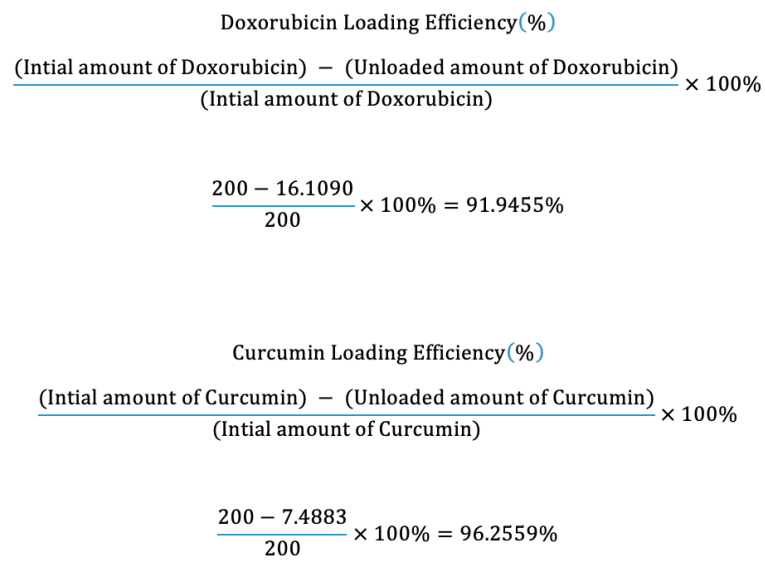

cumin to be $50 \mu \mathrm{g}$ for $5 \mathrm{ppm}, 100 \mu \mathrm{g}$ for $10 \mathrm{ppm}, 200 \mu \mathrm{g}$ for 20 $\mathrm{ppm}, 300 \mu \mathrm{g}$ for $30 \mathrm{ppm}$, and $400 \mu \mathrm{g}$ for $4 \mathrm{ppm}$.

\section{Acknowledgements}

Big thanks for Mr. Tran Duc Thanh for always staying by our side and supporting us the best that he could.

Deepest gratitude to the Vietnam Academy of Science and Technology, Ho Chi Minh City Pedagogical University, and Ho Chi Minh University of Natural Sciences for letting us use your laboratory equipment and space.

\section{References}

1. N. Pradhan, A.Pal, T. Pal (2002), Silver nanoparticle catalyzed reduction of aromatic nitro compounds, Colloid. Surf. A, 196, 247-257.

2. JN Anker, WP Hall, o. Lyandres, NC Shah, J. Zhao, RP Van Duyne (2008), Biosensing with plasmonic nanosensors, Nat. Mater., 7, 442-453.

3. KS Lee, MA El-Sayed (2006), Gold and silver nanoparticles in sensing and imaging: sensitivity of plasmon response to size, shape, and metal composition, J. Phys. Chem. B, 110,19220-19225.

4. KH Cho, JE Park, T. Osaka, SG Park (2005), The study of antimicrobial activity and preservative effects of nanosilver ingredients, Electrochim. Acta, 51,956-960.

5. DK Lee and YS Kang, 2004. Synthesis of silver nanocrystallites by a new thermal decomposition method and their characterization. ETRI Journal, 26 (3), 252-256.

6. B. Taneja, B. Ayyub, and R. Chandra, 2002. Size dependence of the optical spectrum in nanocrystalline silver. Physical Review B, 65, 1-6.

7. Zhang, RC Patel, R. Kothari, CP Johnson, SE Friberg, PA

Aikens (2000), Stable silver clusters and nanoparticles prepared in polyacrylate and inverse micellar solutions, J. Phys. Chem. B, 104,1176-1182.

8. GJ Lee, SI Shin, YC Kim, SG oh (2004), Preparation of silver nanorods through the control of temperature and $p H$ of reaction medium, Mater. Chem. Phys., 84,197-204.

9. Lê Thi My Hanh (2017), Nghiên cu'u bào che nano doxorubicin đa chu'c năng.

10. Elbaz, Nancy \& Ziko, Laila \& Siam, Rania \& Mamdouh, Wael. (2016). Core-Shell Silver/Polymeric NanoparticlesBased Combinatorial Therapy against Breast Cancer In-vitro OPEN. Scientific Reports. 6.10.1038.

11. Patra, J. K., Das, G., Fraceto, L. F., Campos, E. V. R., Rodriguez-Torres, M. del P., Acosta-Torres, L. S.,Shin, H.-S. (2018). Nano based drug delivery systems: recent developments and future prospects. Journal of Nanobiotechnology, 16(1).

12. Rahmani AH, Alsahli MA, Aly SM, Khan MA, Aldebasi YH (2018). Role of Curcumin in Disease Prevention and Treatment. Adv Biomed Res, 7:38.

13. Yin, I. X., Zhang, J., Zhao, I. S., Mei, M. L., Li, Q., \& Chu, C. H. (2020). The Antibacterial Mechanism of Silver Nanoparticles and Its Application in Dentistry. International journal of nanomedicine, 15, 2555-2562.

14. Pfeiffer C, Rehbock C, Hühn D, Carolina Carrillo-Carrion, Dorleta Jimenez de Aberasturi, Vivian Merk, Stephan Barcikowski, Wolfgang J Parak (2014). Interaction of colloidal nanoparticles with their local environment: the (ionic) nanoenvironment around nanoparticles is different from bulk and determines the physico-chemical properties of the nanoparticles. J R Soc Interface. 2014;11(96):20130931.

15. Gaaz TS, Sulong AB, Akhtar MN, Kadhum AA, Mohamad AB, Al-Amiery AA (2015). Properties and Applications of Polyvinyl Alcohol, Halloysite Nanotubes and Their Nanocomposites. Molecules. 2015;20(12):22833-22847.

16. Du Y, Xia L, Jo A, Davis RM, Bissel P, Ehrich MF, Kingston DGI. Synthesis and Evaluation of DoxorubicinLoaded Gold Nanoparticles for Tumor-Targeted Drug Delivery. Bioconjug Chem. 2018 Feb 21;29(2):420-430.

17. Ravindra, S., A. Mulaba-Bafubiandi, V. Rajinkanth, K. Varaprasad, N. Reddy, K. Mohana Raju. Development and Characterization of Curcumin Loaded Silver Nanoparticle Hydrogels for Antibacterial and Drug Delivery Applications. Journal of Inorganic and Organometallic Polymers and Materials 22 (2012): 1254-1262.

18. Mahmudin, L., Suharyadi, E., Utomo, A. B. S., \& Abraha, K. (2016). Influence of stabilizing agent and synthesis 
temperature on the optical properties of silver nanoparticles as active materials in surface plasmon resonance (SPR)

biosensor.

19. Kyrychenko, A., Pasko, D. A., \& Kalugin, O. N. (2017).

Poly(vinyl alcohol) as a water protecting agent for silver nanoparticles: the role of polymer size and structure.

Physical Chemistry Chemical

20. K.A. Juby, Charu Dwivedi, Manmohan Kumar, Swathi Kota, H.S. Misra, P.N. Bajaj, Silver nanoparticle-loaded PVA/gum acacia hydrogel: Synthesis, characterization and antibacterial study, Carbohydrate Polymers, Volume 89, Issue 3, 2012, Pages 906-913.

21. Khanna, P. K., Singh, N., Charan, S., Subbarao, V. V. V. S., Gokhale, R., \& Mulik, U. P. (2005). Synthesis and characterization of $A g / P V A$ nanocomposite by chemical reduction method. Materials Chemistry and Physics, 93(1), 117-121.

22. Kayal, S., \& Ramanujan, R. V. (2010). Doxorubicin loaded $P V A$ coated iron oxide nanoparticles for targeted drug delivery. Materials Science and Engineering: C, 30(3), 484-490.

23. Papadimitriou, S., \& Bikiaris, D. (2009). Novel selfassembled core-shell nanoparticles based on crystalline amorphous moieties of aliphatic copolyesters for efficient controlled drug release. Journal of Controlled Release, 138(2), 177-184.

Author

Nhi Nguyen, the main contributor and translator of this research paper, is a senior at Tran Dai Nghia High School. She has a consuming passion for chemistry and loves nothing more than working in her kitchen and her lab. Her intended major is Chemical Engineering. 